Vol. 76 (2007) [263-268]

\title{
ON GENERALISED PRIME ESSENTIAL RINGS AND SPECIAL AND NONSPECIAL RADICALS
}

\author{
HaLina France-Jackson
}

\begin{abstract}
For a supernilpotent radical $\alpha$ and a special class $\sigma$ of rings we call a ring $R(\alpha, \sigma)$ essential if $R$ is $\alpha$-semisimple and for each ideal $P$ of $R$ with $R / P \in \sigma, P \cap I \neq 0$ whenever $I$ is a nonzero two-sided ideal of $R$. $(\alpha, \sigma)$-essential rings form a generalisation of prime essential rings introduced by $L$. H. Rowen in his study of semiprime rings and their subdirect decompositions and they have been a subject of investigations of many prominent authors since. We show that many important results concerning prime essential rings are also valid for $(\alpha, \sigma)$-essential rings and demonstrate how $(\alpha, \sigma)$-essential rings can be used to determine whether a supernilpotent radical is special. We construct infinitely many supernilpotent nonspecial radicals whose semisimple class of prime rings is zero and show that such radicals form a sublattice of the lattice of all supernilpotent radicals. This generalises Yu.M. Ryabukhin's example.
\end{abstract}

\section{INTRODUCTION}

In this paper all rings are associative and all classes of rings are closed under isomorphisms and contain the one-element ring 0 . The fundamental definitions and properties of radicals can be found in [?] and [?]. If $\mu$ is a hereditary class of rings, $\mathcal{U}(\mu)$ denotes the upper radical generated by $\mu$, that is, the class of all rings which have no nonzero homomorphic images in $\mu$. For any class $\mu$ of rings an ideal $I$ of a ring $R$ is called an $\mu$-ideal if the factor ring $R / I$ is in $\mu$. For a radical $\rho$ the class of all $\rho$-semisimple rings is denoted by $\mathcal{S}(\rho) . \pi$ denotes the class of all prime rings and $\beta$ the prime radical. The notation $I \triangleleft R$ means that $I$ is a two-sided ideal of a ring $R$. For $I \triangleleft R \in \mathcal{S}(\beta)$, $\{r \in R: r I=0\}=\{r \in R: I r=0\}$ is an ideal of $R$ which we shall denote by $I^{*}$. An ideal $I$ of a ring $R$ is called essential in $R$ if $I \cap J \neq 0$ for any nonzero two-sided ideal $J$ of $R$. If $R \in \mathcal{S}(\beta)$ this is equivalent to $I^{*}=0$. 0 is an inessential ideal. Hereditary and essentially closed class of prime rings (respectively semiprime rings) is called a special class (respectively weakly special class) and the upper radical generated by a special class (respectively weakly special class) is called a special radical (respecively supernilpotent radical). Unless otherwise stated, throughout this paper the letter $\alpha$ denotes a supernilpotent radical and $\sigma$ denotes a special class of rings.

Received 5th February, 2007

Copyright Clearance Centre, Inc. Serial-fee code: 0004-9727/07 \$A2.00+0.00. 
A ring $R$ is called ( $\alpha, \sigma)$-essential if $R$ is $\alpha$-semisimple and each $\sigma$-ideal $P$ of $R$ is essential in $R$. $(\alpha, \sigma)$-essential rings form a generalisation of prime essential rings, that is, semiprime rings whose every $\pi$-ideal is essential. Prime essential rings were first introduced by Rowen [?] in his study of semiprime rings and their subdirect decompositions and they have been a subject of investigations of many authors (see [?, ?, ?, ?]) since.

In this paper we show that many important results concerning prime essential rings are also valid for $(\alpha, \sigma)$-essential rings and demonstrate how $(\alpha, \sigma)$-essential rings can be used to determine whether a supernilpotent radical is special. Using $(\alpha, \sigma)$-essential rings, we generalise Ryabukhin's example of supernilpotent nonspecial radical by constructing infinitely many supernilpotent nonspecial radicals $\alpha$ such that $\mathcal{S}(\alpha) \cap \pi=\{0\}$ and show that such radicals form a sublattice of the lattice of all supernilpotent radicals.

\section{MAIN RESUlts}

We start with a useful result which is a generalisation of [?, Proposition 1].

Proposition 1. A ring $R$ is $(\alpha, \sigma)$-essential if and only if $R \in \mathcal{E}_{(\alpha, \sigma)}$, where $\mathcal{E}_{(\alpha, \sigma)}=\{R \in \mathcal{S}(\alpha): 0 \neq I \triangleleft R \Rightarrow I \notin \sigma\}$.

Proof: First assume that $R$ is $(\alpha, \sigma)$-essential and let $0 \neq I \triangleleft R$. Then $R \in \mathcal{S}(\alpha)$ and so $R \in \mathcal{S}(\beta)$ because $\beta \subseteq \alpha$ since $\alpha$ is supernilpotent. If $I \in \sigma$, then $R / I^{*} \in \sigma$ because $\sigma$ is a special class. But then $I^{*}$ is not essential in $R$ because $I^{*} \cap I=0$ which contradicts the assumption that $R$ is $(\alpha, \sigma)$-essential. Thus $I \notin \sigma$ which shows that $R \in \mathcal{E}_{(a, \sigma)}$.

Conversely, assume that $R \in \mathcal{E}_{(\alpha, \sigma)}$ and suppose $R$ contains an inessential $\sigma$-ideal I. Then $0 \neq I^{*} \simeq\left(I+I^{*}\right) / I \triangleleft R / I \in \sigma$ from which it follows that $I^{*} \in \sigma$ since $\sigma$ is hereditary. But this contradicts our assumption that $R \in \mathcal{E}_{(\alpha, \sigma)}$.

Our next result generalises most of [?, Theorem 1].

Theorem 2. $\mathcal{E}_{(\alpha, \sigma)}$ is a weakly special class closed under extensions. Thus $\mathcal{U}\left(\mathcal{E}_{(\alpha, \sigma)}\right)$ is a supernilpotent radical.

Proof: Let $\mu=\mathcal{E}_{(\alpha, \sigma)}$. Since $\alpha$ is supernilpotent, $\mathcal{S}(\alpha) \subseteq \mathcal{S}(\beta)$ so $\mu \subseteq \mathcal{S}(\beta)$. To show hereditariness of $\mu$, let $I \triangleleft R \in \mu$. Then $R \in \mathcal{S}(\alpha)$ and, since $\mathcal{S}(\alpha)$ is hereditary, it follows that $I \in \mathcal{S}(\alpha)$. Suppose $0 \neq J \triangleleft I$ and $J \in \sigma$. Consider the ideal $J_{R}$ of $R$ generated by $J$. As $\mathcal{S}(\alpha) \subseteq \mathcal{S}(\beta)$, it follows that $0 \neq J_{R}^{3} \triangleleft J \in \sigma$ which implies that $J_{R}^{3} \in \sigma$ because $\sigma$ is hereditary. But this means that $R \notin \mu$, a contradiction. Thus $J \notin \sigma$ and so $I \in \mu$.

To show that $\mu$ is closed under essential extensions, let $I \in \mu$ be an essential ideal of a ring $R$. Then $I \in \mathcal{S}(\alpha)$ and it follows that $R \in \mathcal{S}(\alpha)$ because $\mathcal{S}(\alpha)$ is essentially closed. Now, let $0 \neq J \triangleleft R$ and suppose $J \in \sigma$. Then, since $I \cap J \triangleleft J$ and $\sigma$ is hereditary, it follows that $I \cap J \in \sigma$. But, since $I$ is an essential ideal of $R$, it follows that $I \cap J \neq 0$. 
So, since $I \cap J \triangleleft I \in \mu, I \cap J \notin \sigma$, a contradiction. Thus $J \notin \sigma$ which implies that $R \in \mu$ and proves that $\mu$ is closed under essential extensions.

To show that $\mu$ is closed under extensions, let $I$ and $R / I$ be both in $\mu$. Then $I$ and $R / I$ are both in $\mathcal{S}(\alpha)$ and, since $\mathcal{S}(\alpha)$ is closed under extensions, it follows that $R \in \mathcal{S}(\alpha)$. Let $0 \neq J \triangleleft R$. If $I \cap J=0$, then $J \simeq(J+I) / I \triangleleft R / I$ and, since $R / I \in \mu$, it follows that $J \notin \sigma$. If $I \cap J \neq 0$, then $I \cap J \notin \sigma$ because $I \cap J \triangleleft I \in \mu$. Then $J \notin \sigma$ because $I \cap J \triangleleft J$ and $\sigma$ is hereditary. Thus $R \in \mu$ which shows that $\mu$ is closed under extensions.

Since $\mu$ is a hereditary and essentially closed class of semiprime rings, it is weakly special and therefore $\mathcal{U}(\mu)$ is a supernilpotent radical.

Corollary 3. If $\sigma$ contains a nonzero ring $R$ such that $R / I \in \mathcal{S}(\beta)$ implies $R / I \in \sigma$ for every ideal $I \neq R$, then $\mathcal{U}\left(\mathcal{E}_{(\beta, \sigma)}\right)$ is a supernilpotent radical that is not special.

Proof: Since $\beta$ is a supernilpotent radical, it follows from Theorem 2 that $\mathcal{U}\left(\mathcal{E}_{(\beta, \sigma)}\right)$ is supernilpotent. Now, $\mathcal{U}\left(\mathcal{E}_{(\beta, \sigma)}\right) \neq \beta$ because any nonzero ring $R \in \sigma$ such that $R / I \in \mathcal{S}(\beta)$ implies $R / I \in \sigma$ for every ideal $I \neq R$ belongs to $\mathcal{U}\left(\mathcal{E}_{(\beta, \sigma)}\right) \cap \mathcal{S}(\beta)$. Moreover, the class $\mathcal{E}_{(\beta, \sigma)} \subseteq \mathcal{S}\left(\mathcal{U}\left(\mathcal{E}_{(\beta, \sigma)}\right)\right)$ contains all prime essential rings since otherwise some prime essential ring $S$ would contain a nonzero ideal $J \in \sigma \subseteq \pi$ which is impossible. Since every radical $\rho \neq \beta$ whose semisimple class contains all prime essential rings is nonspecial by $[2$, Theorem 1$], \mathcal{U}\left(\mathcal{E}_{(\beta, \sigma)}\right)$ is not a special radical.

COROLlary 4. ([3, Theorem 9(a)]) For the class $\mathcal{E}$ of all prime essential rings the radical $\mathcal{U}(\mathcal{E})$ is supernilpotent but not special.

Proof: Since $\mathcal{E}=\mathcal{E}_{(\beta, \pi)}$ and, as $\beta$ is a supernilpotent radical and $\pi$ is a special class, it follows from Theorem 2 that $\mathcal{U}(\mathcal{E})$ is supernilpotent. Taking $\alpha=\beta, \sigma=\pi$ and any nonzero simple prime ring for $R$ in Corollary 3, we obtain the nonspeciality of $\mathcal{U}(\mathcal{E})$.

LEMMA 5. If $\mu$ is a nonzero weakly special class of rings with $\mathcal{S}(\mathcal{U}(\mu)) \cap \pi=0$, then $\mathcal{U}(\mu)$ is a supernilpotent nonspecial radical. However, the converse is not true.

Proof: Since $\mu$ is a weakly special class, $\mathcal{U}(\mu)$ is supernilpotent. If $\mathcal{U}(\mu)$ were a special radical, then $\mathcal{U}(\mu)=\mathcal{U}(\mathcal{S}(\mathcal{U}(\mu)) \cap \pi)=\mathcal{U}(\{0\})$ would be the class of all rings but this is impossible since $\{0\} \neq \mu \subseteq \mathcal{S}(\mathcal{U}(\mu))$. Thus $\mathcal{U}(\mu)$ is not a special radical.

EXAMPLE 6. For a natural number $n>1$, let $\mathcal{D}_{n}$ be the class of all rings which are subdirect sums of copies of the $n \times n$ matrix ring $M_{n}\left(\mathbf{Z}_{2}\right)$ over $\mathbf{Z}_{2}$ but which have no ideals isomorphic to $M_{n}\left(Z_{2}\right)$ and let $\mathcal{F}$ be the class of all fields. Then $\rho=\mathcal{U}\left(\mathcal{D}_{n} \cup \mathcal{F}\right)$ is a nonspecial radical by [3, Theorem 9 (c)] but $\mathcal{S}(\rho) \cap \pi \neq\{0\}$ because any field $F$ is in $\mathcal{F} \subseteq \mathcal{S}(\rho) \cap \pi$.

Let $F$ be a finite field. For an integer $n \geqslant 1$ let $M_{n}(F)$ be the ring of all $n \times n$ matrices over $F$. It was shown in [5, Lemma 7] that $\mu=\left\{M_{n}(F)\right\}$ is a special class with 
$\mu=\mathcal{S}(\mathcal{U}(\mu)) \cap \pi$.

THEOREM 7. Let $\sigma$ be any class of simple rings with unity such that $\sigma=\mathcal{S}(\mathcal{U}(\sigma)) \cap \pi$. Then $\rho=\mathcal{U}\left(\mathcal{E}_{(\mathcal{U}(\sigma), \sigma)}\right)$ is a supernilpotent nonspecial radical with $\mathcal{S}(\rho) \cap \pi=\{0\}$. Moreover, $\mathcal{S}(\mathcal{U}(\sigma)) \cap \mathcal{E}=\mathcal{E}_{(u(\sigma), \sigma)}$.

Proof: Since $\sigma$ is a special class, it is weakly special. Hence $\alpha=\mathcal{U}(\sigma)$ is a supernilpotent radical and then Theorem 2 implies that so is $\rho$.

To show that $\mathcal{S}(\rho) \cap \pi=0$, suppose $0 \neq R \in \mathcal{S}(\rho) \cap \pi$. Then $R \in \mathcal{S}(\rho)$ and, since the class $\mathcal{E}_{(u(\sigma), \sigma)}$ is weakly special by Theorem $2, R$ is a subdirect sum of rings $R_{i} \in \mathcal{E}_{(U(\sigma), \sigma} \subseteq \mathcal{S}(\mathcal{U}(\sigma))$. Then, as $\mathcal{S}(\mathcal{U}(\sigma))$ is subdirectly closed, it follows that $R \in \mathcal{S}(\mathcal{U}(\sigma)) \cap \pi$. Thus $R \in \sigma$ since $\sigma=\mathcal{S}(\mathcal{U}(\sigma)) \cap \pi$. This implies that $R$ is a simple ring with unity and $R \notin \mathcal{E}_{(U(\sigma), \sigma)}$. But $R$ is also a subdirect sum of rings $R_{i} \in \mathcal{E}_{(u(\sigma), \sigma)}$. So there exist ideals $I_{i}$ of $R$ such that $\cap I_{i}=0$ and $R / I_{i} \simeq R_{i}$ for all $i$. If all $I_{i}=R$, then $R=0$, a contradiction. Thus some $I_{i} \neq R$ and then $I_{i}=0$ since $R$ is a simple ring. But this implies that $R \in \mathcal{E}_{(u(\sigma), \sigma)}$, a contradiction. Thus $\mathcal{S}(\rho) \cap \pi=0$.

Now, since $\{0\} \neq \sigma=\mathcal{S}(\mathcal{U}(\sigma)) \cap \pi$, there exists a nonzero semiprime and $\mathcal{U}(\sigma)$ semisimple ring and hence prime essential $\mathcal{U}(\sigma)$-semisimple ring $S \neq 0$ by [3, Remark 4]. Then $S \in \mathcal{E}_{(u(\sigma), \sigma)}$ because otherwise $S$ would contain a nonzero ideal $I \in \sigma$ and, since $\sigma \subseteq \pi$, this would show that $S$ is not prime essential, a contradiction. This and Theorem 2 imply that $\mathcal{E}_{(u(\sigma), \sigma)}$ is a nonzero weakly special class and, as $\mathcal{S}(\rho) \cap \pi=0$, it follows from Lemma 5 that $\rho$ is not a special radical.

To show that $\mathcal{S}(\mathcal{U}(\sigma)) \cap \mathcal{E}=\mathcal{E}_{(U(\sigma), \sigma)}$, first suppose $0 \neq R \in \mathcal{E}_{(U(\sigma), \sigma)}$ and $R \notin \mathcal{E}$. Then $R \in \mathcal{S}(\mathcal{U}(\sigma))$ and $R$ contains a nonzero ideal $I \in \pi$. But, as $\mathcal{S}(\mathcal{U}(\sigma))$ is hereditary, this implies that $I \in \mathcal{S}(\mathcal{U}(\sigma)) \cap \pi=\sigma$ which implies that $R \notin \mathcal{E}_{(U(\sigma), \sigma)}$, a contradiction. Thus $\mathcal{E}_{(\mathcal{U}(\sigma), \sigma)} \subseteq \mathcal{S}(\mathcal{U}(\sigma)) \cap \mathcal{E}$. Now, if the inclusion were strict, then a ring $S \in \mathcal{S}(\mathcal{U}(\sigma)) \cap \mathcal{E}$ containing a nonzero ideal $J \in \sigma$ could be found and then, since $\sigma \subseteq \pi$, we would get $S \notin \mathcal{E}$, a contradiction. Thus $\mathcal{S}(\mathcal{U}(\sigma)) \cap \mathcal{E}=\mathcal{E}_{U(\sigma), \sigma)}$.

We shall now build infinitely many supernilpotent nonspecial radicals $\rho$ with $\mathcal{S}(\rho)$ $\cap \pi=\{0\}$.

Corollary 8. Let $n \geqslant 1$ be an integer and let $p$ be any prime number. Let $\sigma_{p}=\left\{M_{n}\left(\mathbb{Z}_{p}\right)\right\}$. Then $\rho_{p}=\mathcal{U}\left(\mathcal{E}_{\left(\mathcal{U}\left(\sigma_{p}\right), \sigma_{p}\right)}\right)$ is a supernilpotent nonspecial radical with $\mathcal{S}\left(\rho_{p}\right) \cap \pi=\{0\}$.

In particular, taking $n=1$ and $p=2$ in Corollary 8 , we obtain

COROLLARY 9. ([8]) If $\mathcal{D}$ is the class of all rings which are subdirect sums of copies of $\mathbf{Z}_{2}$ but which have no ideals isomorphic to $\mathbf{Z}_{2}$, then the radical $\rho=\mathcal{U}(\mathcal{D})$ is supernilpotent nonspecial with $\mathcal{S}(\rho) \cap \pi=\{0\}$.

THEOREM 10. The collection $\mathbf{N}$ of all supernilpotent radicals $\rho$ with $\mathcal{S}(\rho) \cap \pi$ $=\{0\}$ is a sublattice of the lattice $\mathbf{K}$ of all supernilpotent radicals. 
Proof: Let $\rho, \gamma \in \mathbb{N}$. Since $\mathcal{S}(\rho \vee \gamma)=\mathcal{S}(\rho) \cap \mathcal{S}(\gamma)$, it follows that $\mathcal{S}(\rho \vee \gamma) \cap \pi=\{0\}$ which implies that $\rho \vee \gamma \in \mathbb{N}$.

To show that $\rho \wedge \gamma \in \mathbb{N}$, suppose $0 \neq R \in \mathcal{S}(\rho \wedge \gamma) \cap \pi$. If the $\rho$-radical $\rho(R)$ of $R$ is zero, then, since $R \in \pi$, it follows that $R \in \mathcal{S}(\rho) \cap \pi$ which is impossible since $\mathcal{S}(\rho) \cap \pi=\{0\}$ because $\rho \in \mathbf{N}$. Thus $\rho(R) \neq 0$ and, similarly, $\gamma(R) \neq 0$. Now, since $\rho(R)$ and $\gamma(R)$ are nonzero ideals of $R$ and $R$ is a prime ring, it follows that $0 \neq \rho(R)$ $\gamma(R) \triangleleft R$. But, since both $\rho$ and $\gamma$ are hereditary and $\rho(R) \gamma(R) \triangleleft \rho(R) \in \rho$ and $\rho(R)$ $\gamma(R) \triangleleft \gamma(R) \in \gamma$, it then follows that $\rho(R) \gamma(R) \in \rho \wedge \gamma$ which contradicts the fact that $R \in \mathcal{S}(\rho \wedge \gamma)$. Thus $\mathcal{S}(\rho \wedge \gamma) \cap \pi=\{0\}$ which implies that $\rho \wedge \gamma \in \mathbb{N}$.

REMark 11. We do not know whether $\mathbb{N}$ is a complete sublattice of the lattice $\mathbb{K}$. To answer this question in the negative, it would suffice to show that $\wedge \rho_{p} \notin \mathbb{N}$, where $\rho_{p}$ are described in Corollary 8.

In what follows for any ring $R$ let $\Delta_{\sigma}=\cap\left\{I: I\right.$ is a $\sigma$-ideal of $R$ with $\left.I^{*} \neq 0\right\}$. If $I^{*}=0$ for all $\sigma$-ideals $I$ of $R$, we take $\Delta_{\sigma}=R$.

LEMMA 12. Any ring $R \in \mathcal{S}(\alpha)$ is a subdirect sum of $R / \Delta_{\sigma}$ and $R / \Delta_{\sigma}^{*}$. Moreover, $R / \Delta_{\sigma}^{*} \in \mathcal{E}_{(\boldsymbol{\alpha}, \boldsymbol{\sigma})}$.

Proof: Since $\Delta_{\sigma} \cap \Delta_{\sigma}^{*}=0$, it suffices to show that $R / \Delta_{\sigma}^{*} \in \mathcal{E}_{(\alpha, \sigma)}$.

Now, since $\Delta_{\sigma} \triangleleft R \in \mathcal{S}(\alpha)$ and $\mathcal{S}(\alpha)$ is hereditary, it follows that $\Delta_{\sigma} \in \mathcal{S}(\alpha)$. Now, if $\Delta_{\sigma}=0$, then $R / \Delta_{\sigma}^{*}=0 \in \mathcal{E}_{(a, \sigma)}$ and we are done. So assume that $\Delta_{\sigma} \neq 0$. Then the factor ring $R / \Delta_{\sigma}^{*}$ is in $\mathcal{S}(\alpha)$ since it has an essential ideal $\left(\Delta_{\sigma}+\Delta_{\sigma}^{*}\right) / \Delta_{\sigma}^{*} \simeq \Delta_{\sigma} \in \mathcal{S}(\alpha)$ and $\mathcal{S}(\alpha)$ is essentially closed. Let $\bar{A}=A / \Delta_{\sigma}^{*}$ be a $\sigma$-ideal of $\bar{R}=R / \Delta_{\sigma}^{*}$ so that $R / A \in \sigma$. For any ideal $\bar{A} \prime=A / / \Delta_{\sigma}^{*}$ of $\bar{R}$ such that $\bar{A} / \bar{A}=0$, we have $A / A \subseteq \Delta_{\sigma}^{*}$ so $\Delta_{\sigma} A \prime A \subseteq \Delta_{\sigma} \Delta_{\sigma}^{*}=0$. It suffices to show that $\Delta_{\sigma} A \prime=0$ since then $A^{\prime} \subseteq \Delta_{\sigma}^{*}$ implying $\bar{A} \prime=\overline{0}$ in $\bar{R}$. If $A$ is essential in $R$, then $\Delta_{\sigma} A \prime A=0$ implies $\Delta_{\sigma} A \prime=0$ and we are done. If $A$ is inessential in $R$, then $\Delta_{\sigma} \subseteq A$, so $\left(\Delta_{\sigma} A \prime\right)^{2} \subseteq \Delta_{\sigma} A \prime \Delta_{\sigma} \subseteq \Delta_{\sigma} A \prime A=0$; hence $\Delta_{\sigma} A \prime=0$ since $R \in \mathcal{S}(\alpha) \subseteq \mathcal{S}(\beta)$. Then $\bar{A}$ is essential in $\bar{R}$ which implies that $\bar{R}$ $\in \mathcal{E}_{(\alpha, \sigma)}$.

A supernilpotent radical $\rho$ is special if every $R \in \mathcal{S}(\rho)$ is a subdirect sum of rings in $\mathcal{S}(\rho) \cap \pi$.

THEOREM 13. A supernilpotent radical $\rho$ that contains $\alpha$ is special if and only if every ring $A \in \mathcal{E}_{(\alpha, \sigma)} \cap \mathcal{S}(\rho)$ is a subdirect sum of rings in $\mathcal{S}(\rho) \cap \pi$.

Proof: If $\rho$ is a special radical, then $\mathcal{S}(\rho) \cap \pi$ is a special class and $\rho=\mathcal{U}(\mathcal{S}(\rho) \cap \pi)$; hence the result follows.

Conversely, let $A \in \mathcal{S}(\rho)$. We need to show that $A$ is a subdirect sum of rings in $\mathcal{S}(\rho) \cap \pi$. If $P^{*}=0$ for all $\sigma$-ideals $P$ of $A$, then Proposition 1 implies that $A \in \mathcal{E}_{(\alpha, \sigma)} \cap \mathcal{S}(\rho)$ and the result follows from the assumption. If $P^{*} \neq 0$, then $A / P \in \mathcal{S}(\rho)$ since it has an essential ideal $\left(P^{*}+P\right) / P \simeq P^{*} \in \mathcal{S}(\rho)$ and $\mathcal{S}(\rho)$ is essentially closed. But $A / P \in \sigma \subseteq \pi$ 
so $A / P \in \mathcal{S}(\rho) \cap \pi$ for every $\sigma$-ideal $P$ of $A$ with $P^{*} \neq 0$. Therefore the proof is completed if $\Delta_{\sigma}=0$. So assume that $\Delta_{\sigma} \neq 0$. Then $A / \Delta_{\sigma}^{*} \in \mathcal{S}(\rho)$ because it contains an essential ideal $\left(\Delta_{\sigma}+\Delta_{\sigma}^{*}\right) / \Delta_{\sigma}^{*} \simeq \Delta_{\sigma} \in \mathcal{S}(\rho)$ and $\mathcal{S}(\rho)$ is hereditary and essentially closed. But, as $A \in \mathcal{S}(\rho) \subseteq \mathcal{S}(\alpha)$ Lemma 12 implies that $A$ is a subdirect sum of $A / \Delta_{\sigma}$ and $A / \Delta_{\sigma}^{*} \in \mathcal{E}_{(\alpha, \sigma)}$. But then $A / \Delta_{\sigma}^{*} \in \mathcal{S}(\rho) \cap \mathcal{E}_{(\alpha, \sigma)}$ and it follows from the assumption that $A / \Delta_{\sigma}^{*}$ is a subdirect sum of rings in $\mathcal{S}(\rho) \cap \pi$, that is, there exist ideals $J_{i}$ of $A$ such that $J_{i} / \Delta_{\sigma}^{*} \triangleleft A / \Delta_{\sigma}^{*}, \cap J_{i} \subseteq \Delta_{\sigma}^{*}$ and $A / J_{i} \in \mathcal{S}(\rho) \cap \pi$. But $A / \Delta_{\sigma}$ is also a subdirect sum of rings from $\mathcal{S}(\rho) \cap \pi$ because $\cap\left\{P_{j} / \Delta_{\sigma}: P_{j}\right.$ is a $\sigma$-ideal of $A$ with $\left.P_{j}^{*} \neq 0\right\}=0$ in $A / \Delta_{\sigma}$ and, as it was shown above, $A / P_{j} \in \mathcal{S}(\rho) \cap \pi$ for every $\sigma$-ideal $P_{j}$ of $A$ with $P_{j}^{*} \neq 0$. Thus we get $\left(\cap J_{i}\right) \cap\left(\cap P_{j}\right) \subseteq \Delta_{\sigma}^{*} \cap \Delta_{o}=0$ which shows that $A$ is a subdirect sum of rings in $\mathcal{S}(\rho) \cap \pi$.

Taking $\alpha=\beta$ and $\sigma=\pi$ in Theorem 13, we obtain

COROLlary 14. ([3, Theorem 6]) A supernilpotent radical $\rho$ is special if and only if every prime essential $\rho$-semisimple ring is a subdirect sum of prime $\rho$-semisimple rings.

\section{REFERENCES}

[1] V.A. Andrunakievich and Yu.M. Ryabukhin, Radicals of algebras and structure theory, (Russian) (Nauka, Moscow, 1979).

[2] H. France-Jackson, 'On prime essential rings', Bull. Austral. Math. Soc. 47 (1993), 287-290.

[3] B.J. Gardner and P.N. Stewart, 'Prime essential rings', Proc. Edinburgh Math. Soc. 34 (1991), 241-250.

[4] B.J. Gardner and R. Wiegandt, Radical theory of rings (Marcel Dekker, New York, 2004).

[5] K.K. Krachilov, 'Coatoms of the lattice of special radicals', (Russian), Mat. Issled. 49 (1979), 80-86.

[6] E. Matlis, 'The minimal prime spectrum of a reduced ring', Mlinois J. Math. 27 (1983), 353-391.

[7] L.H. Rowen, 'A subdirect decomposition of semiprime rings and its application to maximal quotient rings', Proc. Amer. Math. Soc. 46 (1974), 176-180.

[8] Yu.M. Ryabukhin, 'Overnilpotent and special radicals', Studies in Algebra and Math. Anal., Izdat "Karta Moldovenjaske" Kishinev (1965), 65-72.

[9] H. Zand, 'A note on prime essential rings', Bull. Austral. Math. Soc. 49 (1994), 55-57.

Department of Mathematics and Applied Mathematics

Summerstrand Campus (South)

PO Box 77000

Nelson Mandela Metropolitan University

Port Elizabeth 6031

South Africa

e-mail: cbf@easterncape.co.uk 